\title{
Pattern Avoidance and Dominating Compositions
}

\author{
Krishna Menon and Anurag Singh \\ Department of Mathematics, Chennai Mathematical Institute, India 603103 \\ Email: krishnamenon@cmi.ac.in, anuragsingh@cmi.ac.in \\ Received: April 16, 2021, Accepted: July 30, 2021, Published: August 6, 2021 \\ The authors: Released under the CC BY-ND license (International 4.0)
}

ABstract: Jelínek, Mansour, and Shattuck studied Wilf-equivalence among pairs of patterns of the form $\{\sigma, \tau\}$ where $\sigma$ is a set partition of size 3 with at least two blocks. They obtained an upper bound for the number of Wilf-equivalence classes for such pairs. We show that their upper bound is the exact number of equivalence classes, thus solving a problem posed by them.

Keywords: Dominating equivalent compositions; Pattern avoidance; Set partition; Wilf-equivalence

2020 Mathematics Subject Classification: 05A15; 05A18; 05A19

\section{Introduction}

For any $n \geq 1$, a partition of $[n]=\{1, \ldots, n\}$ is a collection of disjoint nonempty subsets of $[n]$ whose union is $[n]$. The most common method of representing a set partition given by $\left\{B_{1}, B_{2}, \ldots, B_{k}\right\}$ is to write the blocks as

$$
B_{1} / B_{2} / \cdots / B_{k} \text {. }
$$

The usual convention followed is to order the blocks such that

$$
\min \left(B_{1}\right)<\min \left(B_{2}\right)<\cdots<\min \left(B_{k}\right) .
$$

Sometimes it is also convenient, when it does not cause confusion, to write the elements of each block without braces or commas. For example, the partition $\{\{1,3,4\},\{2,6\},\{5,7,8\}\}$ of $[8]$ is written as $\{1,3,4\} /\{2,6\} /\{5,7,8\}$ or $134 / 26 / 578$.

A partition $B_{1} / B_{2} / \cdots / B_{k}$ of $[n]$ such that $\min \left(B_{1}\right)<\min \left(B_{2}\right)<\cdots<\min \left(B_{k}\right)$ can be represented by the sequence $a_{1} a_{2} \cdots a_{n}$ where $a_{i}=j$ if $i \in B_{j}$. This sequence is called the restricted growth function associated with the partition. For example, the partition 134/26/578 has a corresponding sequence 12113233. For more on restricted growth functions, the interested reader is referred to Sagan's paper [7, Section 4]. Henceforth, we will represent partitions using restricted growth functions. The following definition of pattern avoidance in set partitions was first introduced by Sagan [7].

Definition 1.1. A partition $\sigma=\sigma_{1} \sigma_{2} \cdots \sigma_{n}$ contains a partition (or pattern) $\pi=\pi_{1} \pi_{2} \cdots \pi_{m}$ if there exists a subsequence $1 \leq h(1)<h(2)<\cdots<h(m) \leq n$ such that for any $i, j \in[m], \sigma_{h(i)}=\sigma_{h(j)}$ if and only if $\pi_{i}=\pi_{j}$ and $\sigma_{h(i)}<\sigma_{h(j)}$ if and only if $\pi_{i}<\pi_{j}$. If $\sigma$ does not contain $\pi$, we say that $\sigma$ avoids the pattern $\pi$.

The topic of pattern avoidance has been an active area of research in enumerative combinatorics, starting with Knuth's work on permutations [6]. The study of pattern avoidance in set partitions was initiated by Klazar [5]. Since then, several different notions of pattern avoidance of set partitions have been studied (see, e.g., the work of Chen et al. [2], Goyt [3], or Bloom and Elizalde [1]).

Let $T$ be a set of patterns. We will denote the set of partitions of $[n]$ that avoid all the patterns of $T$ as $P_{n}(T)$ and the number of such partitions, i.e., $\left|P_{n}(T)\right|$ as $p_{n}(T)$.

Definition 1.2. Two sets of patterns $T$ and $R$ are said to be Wilf-equivalent, written as $T \sim R$, if $p_{n}(T)=p_{n}(R)$ for all $n \geq 1$. 
For example, $P_{n}(\{123\})$ is the set of partitions of $[n]$ with at most two blocks and $P_{n}(\{122\})$ is the set of partitions where any block not containing 1 is a singleton. Hence we get $\{123\} \sim\{122\}$ since $p_{n}(\{123\})=$ $p_{n}(\{122\})=2^{n-1}$ for all $n \geq 1$.

In this article, our focus will be on pairs of patterns $\{\sigma, \tau\}$, where $\sigma$ is a pattern of size 3 with at least 2 blocks. Such pairs are called $(3, k)$-pairs when the size of $\tau$ is $k$. Jelínek, Mansour, and Shattuck [4] studied such pairs and obtained an upper bound for the number of Wilf-equivalence classes of $(3, k)$-pairs. This was done by describing various Wilf-equivalences among $(3, k)$-pairs. They also showed that any other Wilf-equivalences, if they exist, are between $(3, k)$-pairs of the form $\{112, \tau\}$ and left open the following problem.

Problem ( $[4$, Problem 2.17]). Are there any more equivalences among the $(3, k)$-pairs of the form $\{112, \tau\}$ other than those that we know about? Equivalently, are there any two distinct 2-free integer partitions that are $\stackrel{d}{\sim}$-equivalent (see Definition 2.3)?

The main aim of this article is to answer this question. In Section 2, we describe the results obtained by Jelínek, Mansour, and Shattuck [4], and show how the second question is equivalent to the first in the above problem. In Section 3, we answer this equivalent question and hence prove that there are no other Wilf-equivalences among (3,k)-pairs other than the ones described by Jelínek, Mansour, and Shattuck in [4].

\section{Wilf-equivalences among $(3, k)$-pairs}

In this section, we will state the Wilf-equivalences between $(3, k)$-pairs derived by Jelínek, Mansour, and Shattuck [4].

Note that if we are studying $P_{n}(T)$ where $T=\left\{\tau_{1}, \tau_{2}, \ldots\right\}$, we can assume that $\tau_{i}$ avoids $\tau_{j}$ for all $i \neq j$. For example, when avoiding a $(3, k)$-pair $\{121, \tau\}$, we can assume that $\tau$ avoids 121 . It can be shown that if the number of blocks and size of each block is specified, there is a unique partition that avoids 121 . Namely, if a partition has $m$ blocks such that the $i^{\text {th }}$ block has $a_{i}$ elements for all $i \in[m]$, the unique such partition avoiding 121 is $1^{a_{1}} 2^{a_{2}} \cdots m^{a_{m}}$. Here, $k^{a}$ represents $a$ consecutive copies of $k$.

Definition 2.1. A composition of a positive integer $n$ is a sequence $\left(a_{1}, a_{2}, \ldots, a_{m}\right)$ such that $a_{1}+\cdots+a_{m}=n$. The set of compositions of $n$ is denoted as $C_{n}$.

For example, $C_{3}=\{(1,1,1),(1,2),(2,1),(3)\}$. The above discussion implies that $P_{n}(\{121\})$ is in bijection with $C_{n}$. The partition avoiding 121 associated to the composition $a=\left(a_{1}, \ldots, a_{m}\right)$ is

$$
\tau_{121}(a)=1^{a_{1}} 2^{a_{2}} \cdots m^{a_{m}} .
$$

It can be shown that the partitions of the form $\tau_{121}(b)$ that contains $\tau_{121}(a)$ are those where $b$ has a subsequence, having the same length as $a$, such that each term in the subsequence has a value at least that of the corresponding term in $a$.

Definition 2.2. The composition $b=\left(b_{1}, \ldots, b_{k}\right)$ is said to dominate the composition $a=\left(a_{1}, \ldots, a_{m}\right)$ if there exists a subsequence $1 \leq i(1)<i(2)<\cdots<i(m) \leq k$ such that $b_{i(j)} \geq a_{j}$ for all $j \in[m]$. Such a subsequence is called an occurrence of $a$ in $b$. For any positive integer $n, D_{n}(a)$ will denote the set of compositions of $n$ that dominate a.

Hence, we get that $P_{n}\left(\left\{121, \tau_{121}(a)\right\}\right)=\left\{\tau_{121}(b) \mid b \in C_{n} \backslash D_{n}(a)\right\}$. We now describe the Wilf-equivalences among $(3, k)$-pairs of the form $\left\{121, \tau_{121}(a)\right\}$. From the description of $P_{n}\left(\left\{121, \tau_{121}(a)\right\}\right)$, it is clear that $\left\{121, \tau_{121}(a)\right\} \sim\left\{121, \tau_{121}\left(a^{\prime}\right)\right\}$ if and only if $\left|D_{n}(a)\right|=\left|D_{n}\left(a^{\prime}\right)\right|$ for all $n \geq 1$. Hence we make the following definition.

Definition 2.3. For two compositions a and $a^{\prime}$, we say a and $a^{\prime}$ are dominating equivalent, written as a $\stackrel{d}{\sim} a^{\prime}$, if for all positive integers $n,\left|D_{n}(a)\right|=\left|D_{n}\left(a^{\prime}\right)\right|$.

Therefore, $\left\{121, \tau_{121}(a)\right\} \sim\left\{121, \tau_{121}\left(a^{\prime}\right)\right\}$ if and only if $a \stackrel{d}{\sim} a^{\prime}$. Using similar ideas it can also be shown that $P_{n}(\{112\})$ is in bijection with compositions of $n$, where to a composition $a=\left(a_{1}, \ldots, a_{m}\right)$ we associate the partition

$$
\tau_{112}(a)=12 \cdots(m-1) m m^{a_{m}-1} \cdots 2^{a_{2}-1} 1^{a_{1}-1} .
$$

Also, $P_{n}\left(\left\{112, \tau_{112}(a)\right\}\right)=\left\{\tau_{112}(b) \mid b \in C_{n} \backslash D_{n}(a)\right\}$ and $\left\{112, \tau_{112}(a)\right\} \sim\left\{112, \tau_{112}\left(a^{\prime}\right)\right\}$ if and only if $a \stackrel{d}{\sim} a^{\prime}$.

Using that fact that $p_{k}(\sigma)=2^{k-1}$ for all patterns $\sigma$ of size 3 other than 111 (see [7, Theorem 4.3] for details), we get that if $k<k^{\prime}$ then there are $2^{k-1}$ partitions of size $k$ avoiding a $\left(3, k^{\prime}\right)$-pair but only $2^{k-1}-1$ partitions of size $k$ avoiding a $(3, k)$-pair. Hence, no $(3, k)$-pair can be Wilf-equivalent to a $\left(3, k^{\prime}\right)$-pair where $k \neq k^{\prime}$.

A detailed discussion of the above facts can be found in the paper of Jelínek, Mansour, and Shattuck [4]. In fact, they also showed that all except one Wilf-equivalence class of $(3, k)$-pairs correspond to dominating equivalence classes of $C_{k}$. 
1. The Wilf-equivalence class corresponding to the dominating equivalence class $E$ containing $(1, \ldots, 1)$ consists of the following pairs:

(a) $\left\{121, \tau_{121}(a)\right\}$ where $a \in E$,

(b) $\left\{112, \tau_{112}(a)\right\}$ where $a \in E$,

(c) $\{122, \tau\}$ where $\tau \in P_{k}(\{122\})$, and

(d) $\left\{123, \tau^{\prime}\right\}$ where $\tau^{\prime} \neq 1^{k}$.

2. The Wilf-equivalence class corresponding to a dominating equivalence class $E$ not containing $(1, \ldots, 1)$ consists of the following pairs:

(a) $\left\{121, \tau_{121}(a)\right\}$ where $a \in E$, and

(b) $\left\{112, \tau_{112}(a)\right\}$ where $a \in E$.

3. The pair $\left\{123,1^{k}\right\}$ is not Wilf-equivalent to any other $(3, k)$-pair.

Hence, if we denote the number of dominating equivalence classes in $C_{k}$ as $\xi_{k}$, we get that $(3, k)$-pairs split up into $1+\xi_{k}$ Wilf-equivalence classes. Hence, we now shift our focus to the dominating equivalence among compositions.

\section{Dominating equivalence}

We first recall a few results from the paper of Jelínek, Mansour, and Shattuck [4].

Lemma 3.1 ( $\left[4\right.$, Lemma 2.3]). If $a=\left(a_{1}, \ldots, a_{m}\right)$ and $a^{\prime}=\left(a_{\sigma(1)}, \ldots, a_{\sigma(m)}\right)$ where $\sigma$ is a permutation of $[m]$, then $a \stackrel{d}{\sim} a^{\prime}$.

Lemma 3.2 ( $\left[4\right.$, Lemma 2.4]). If $a=\left(a_{1}, \ldots, a_{m-1}, 2\right)$ and $a^{\prime}=\left(a_{1}, \ldots, a_{m-1}, 1,1\right)$, then $a \stackrel{d}{\sim} a^{\prime}$

These lemmas imply that to each composition we can associate a unique 2-free integer partition which is dominating equivalent to it. This is done by first rearranging the terms in decreasing order and then replacing each 2 in the composition with two 1 s.

Example 3.1. The composition $(3,2,5,1,1,3)$ is converted to the associated unique 2-free integer partition as follows:

$$
(3,2,5,1,1,3) \stackrel{d}{\sim}(5,3,3,2,1,1) \stackrel{d}{\sim}(5,3,3,1,1,1,1)
$$

Hence, any two compositions that correspond to the same 2-free integer partition are dominating equivalent. We will now show that the converse is true as well, which was conjectured by Jelínek, Mansour, and Shattuck [4].

Theorem 3.1. Two compositions a and $a^{\prime}$ are dominating equivalent if and only if they correspond to the same 2-free integer partition.

The above theorem implies that $\xi_{k}$, i.e., the number of dominating equivalence classes in $C_{k}$, is $p(k)-p(k-2)$, where for any $n \geq 1, p(n)$ is the number of integer partitions of $n$. The sequence $\left(\xi_{k}\right)_{k \geq 0}$ is listed in the OEIS [8] as A027336. Hence the number of Wilf-equivalences classes of $(3, k)$-pairs is $p(k)-p(k-2)+1$.

To prove the theorem, we first need a few lemmas and definitions.

Lemma 3.3. If $a \stackrel{d}{\sim} a^{\prime}$ for two compositions $a$ and $a^{\prime}$ of $m$ and $n$ respectively, then $m=n$.

Proof. Suppose to the contrary that $m$ and $n$ are distinct. Without loss of generality, we assume $m<n$. Then $D_{m}(a)=\{a\}$ whereas $D_{m}\left(a^{\prime}\right)$ is an empty set. This contradicts the fact that $a \stackrel{d}{\sim} a^{\prime}$ and hence proves our lemma.

Definition 3.1. The composition $b=\left(b_{1}, \ldots, b_{k}\right)$ is said to h-dominate the composition a if $b$ dominates a and either $k=1$ or $\left(b_{1}, \ldots, b_{k-1}\right)$ does not dominate $a$. For any positive integer $n, D_{n}^{h}(a)$ is the set of compositions of $n$ that $h$-dominate $a$. We say a and $a^{\prime}$ are $h$-dominating equivalent, written as $a \stackrel{h}{\sim} a^{\prime}$, if for all positive integers $n,\left|D_{n}^{h}(a)\right|=\left|D_{n}^{h}\left(a^{\prime}\right)\right|$.

We will now show that dominating equivalence is the same as h-dominating equivalence. Before doing so, we set up some notations. Let $c=\left(c_{1}, \ldots, c_{k}\right)$ and $c^{\prime}=\left(c_{1}^{\prime}, \ldots, c_{k^{\prime}}^{\prime}\right)$ be compositions. We will denote the composition $\left(c_{1}, \ldots, c_{k}, c_{1}^{\prime}, \ldots, c_{k^{\prime}}^{\prime}\right)$ by $\left(c, c^{\prime}\right)$. Similarly, for any positive integer $s$, denote the composition $\left(c_{1}, \ldots, c_{k}, s\right)$ by $(c, s)$. 
Lemma 3.4. For any two compositions $a$ and $a^{\prime}, a \stackrel{d}{\sim} a^{\prime}$ if and only if $a \stackrel{h}{\sim} a^{\prime}$.

Proof. Note that for any composition $a$,

$$
D_{n}(a)=D_{n}^{h}(a) \sqcup \bigsqcup_{i=1}^{n-1}\left\{\left(c, c^{\prime}\right) \mid c \in D_{i}^{h}(a), c^{\prime} \in C_{n-i}\right\} .
$$

This is obtained by finding, for each $b=\left(b_{1}, \ldots, b_{k}\right) \in D_{n}(a)$, the least $j \in[k]$ such that $\left(b_{1}, \ldots, b_{j}\right)$ dominates $a$. This element $b$ of $D_{n}(a)$ belongs to exactly one of the $n$ sets on the right side. If $j=k$, then $b \in D_{n}^{h}(a)$ or else $b \in\left\{\left(c, c^{\prime}\right) \mid c \in D_{i}^{h}(a), c^{\prime} \in C_{n-i}\right\}$ where $i=b_{1}+\cdots+b_{j}$.

Suppose $a \stackrel{h}{\sim} a^{\prime}$. The equality (1) implies that for all positive integers $n$,

$$
\begin{aligned}
\left|D_{n}(a)\right| & =\left|D_{n}^{h}(a)\right|+\sum_{i=1}^{n-1}\left|D_{i}^{h}(a)\right| \cdot\left|C_{n-i}\right| \\
& =\left|D_{n}^{h}\left(a^{\prime}\right)\right|+\sum_{i=1}^{n-1}\left|D_{i}^{h}\left(a^{\prime}\right)\right| \cdot\left|C_{n-i}\right| \\
& =\left|D_{n}\left(a^{\prime}\right)\right| .
\end{aligned}
$$

Hence we get $a \stackrel{d}{\sim} a^{\prime}$.

Conversely, suppose $a \stackrel{d}{\sim} a^{\prime}$. We will prove $\left|D_{n}^{h}(a)\right|=\left|D_{n}^{h}\left(a^{\prime}\right)\right|$ for all $n \geq 1$ by induction on $n$. We have $\left|D_{1}^{h}(a)\right|=\left|D_{1}(a)\right|=\left|D_{1}\left(a^{\prime}\right)\right|=\left|D_{1}^{h}\left(a^{\prime}\right)\right|$. Let $n \geq 2$ and $\left|D_{k}^{h}(a)\right|=\left|D_{k}^{h}\left(a^{\prime}\right)\right|$ for all $k<n$. Using (1), we get

$$
\begin{aligned}
\left|D_{n}^{h}(a)\right| & =\left|D_{n}(a)\right|-\sum_{i=1}^{n-1}\left|D_{i}^{h}(a)\right| \cdot\left|C_{n-i}\right| \\
& =\left|D_{n}\left(a^{\prime}\right)\right|-\sum_{i=1}^{n-1}\left|D_{i}^{h}\left(a^{\prime}\right)\right| \cdot\left|C_{n-i}\right| \\
& =\left|D_{n}^{h}\left(a^{\prime}\right)\right| .
\end{aligned}
$$

Hence we get $a \stackrel{h}{\sim} a^{\prime}$, which completes the proof of the lemma.

Lemma 3.5. If a and $a^{\prime}$ are two compositions and $s$ is a positive integer such that $(a, s) \stackrel{h}{\sim}\left(a^{\prime}, s\right)$, then we have $a \stackrel{h}{\sim} a^{\prime}$

Proof. Let $a$ be a composition and $s$ be a positive integer. Let $C_{k, s}$ denote those compositions of $k$ of the form $\left(c_{1}, \ldots, c_{m}\right)$ where $c_{i}<s$ for all $i \in[m-1]$ and $c_{m} \geq s$. Note that

$$
D_{n}^{h}((a, s))=\bigsqcup_{i=1}^{n-1}\left\{\left(c, c^{\prime}\right) \mid c \in D_{i}^{h}(a), c^{\prime} \in C_{n-i, s}\right\} .
$$

This is obtained by finding, for each $b=\left(b_{1}, \ldots, b_{m}\right) \in D_{n}^{h}((a, s))$, the least $j \in[m]$ such that $\left(b_{1}, \ldots, b_{j}\right)$ dominates $a$. Since $b \in D_{n}^{h}((a, s))$, this would mean that $b_{j+1}, \ldots, b_{m-1}$ are all strictly less than $s$ and $b_{m} \geq s$. Note that $\left|C_{i, s}\right|=0$ for all $i<s$ and that $\left|C_{s, s}\right|=1$. Hence an implication of the equality (2) is that for any positive integer $n$,

$$
\left|D_{n+s}^{h}((a, s))\right|=\left|D_{n}^{h}(a)\right|+\sum_{i=s+1}^{n+s-1}\left|D_{n+s-i}^{h}(a)\right| \cdot\left|C_{i, s}\right| .
$$

Let $a^{\prime}$ be a composition such that $(a, s) \stackrel{h}{\sim}\left(a^{\prime}, s\right)$. We will prove $\left|D_{n}^{h}(a)\right|=\left|D_{n}^{h}\left(a^{\prime}\right)\right|$ for all $n \geq 1$ by induction on $n$. If $\left|D_{1}^{h}(a)\right| \neq\left|D_{1}^{h}\left(a^{\prime}\right)\right|$, we would have exactly one of $a$ or $a^{\prime}$ being the composition of 1 . This would mean $(a, s)$ and $\left(a^{\prime}, s\right)$ are compositions of different numbers, which contradicts Lemma 3.3 (since Lemma 3.4 implies that $\left.(a, s) \stackrel{d}{\sim}\left(a^{\prime}, s\right)\right)$. Let $n \geq 2$ and $\left|D_{k}^{h}(a)\right|=\left|D_{k}^{h}\left(a^{\prime}\right)\right|$ for all $k<n$. From (3), we get

$$
\begin{aligned}
\left|D_{n}^{h}(a)\right| & =\left|D_{n+s}^{h}((a, s))\right|-\sum_{i=s+1}^{n+s-1}\left|D_{n+s-i}^{h}(a)\right| \cdot\left|C_{i, s}\right| \\
& =\left|D_{n+s}^{h}\left(\left(a^{\prime}, s\right)\right)\right|-\sum_{i=s+1}^{n+s-1}\left|D_{n+s-i}^{h}\left(a^{\prime}\right)\right| \cdot\left|C_{i, s}\right| \\
& =\left|D_{n}^{h}\left(a^{\prime}\right)\right| .
\end{aligned}
$$

Hence we get $a \stackrel{h}{\sim} a^{\prime}$, which completes the proof of the lemma. 
Corollary 3.1. If $a$ and $a^{\prime}$ are two compositions and $s$ is a positive integer such that $(a, s) \stackrel{d}{\sim}\left(a^{\prime}, s\right)$, then we have $a \stackrel{d}{\sim} a^{\prime}$.

Proof. Combine Lemma 3.4 and Lemma 3.5.

We can now prove our main theorem.

Proof of Theorem 3.1. We have to show that if $a$ and $b$ are two compositions such that $a \stackrel{d}{\sim} b$, then they correspond to the same 2-free integer partition. By Lemma 3.3, such $a$ and $b$ are compositions of the same number $n$. We will prove the theorem by induction on $n$, the case $n=1$ being trivial. Let $n \geq 2$ and suppose the statement is true for all numbers less than $n$. Suppose $a \stackrel{d}{\sim} b$ are two compositions of $n$. Using Lemma 3.1 and Lemma 3.2, we can assume that $a_{1} \geq \cdots \geq a_{m}$ and $a_{m-1} \geq 2$ and similarly that $b_{1} \geq \cdots \geq b_{k}$ and $b_{k-1} \geq 2$ (this can be done by reordering the terms in decreasing order and then changing any pair of 1's to a 2).

We will now compute $\left|D_{n+1}(a)\right|$. If $a_{m} \geq 2$, we get $\left|D_{n+1}(a)\right|=2 m+1$. This is because any composition in $D_{n+1}(a)$ is obtained by either

1. inserting the term 1 before $a_{1}$, between $a_{i}$ and $a_{i+1}$ for some $i \in[m-1]$, or after $a_{m}$, or

2. adding 1 to some term of $a$.

Since all terms of $a$ are greater than 1 in this case, each method of obtaining a composition of $D_{n+1}(a)$ described above results in a different composition.

When $a_{m}=1$, using the same logic as above but noting that adding 1 before $a_{m}$ and adding 1 after $a_{m}$ both result in the same composition, we get $\left|D_{n+1}(a)\right|=2 m$.

Using similar arguments for $b$ and since we must have $\left|D_{n+1}(a)\right|=\left|D_{n+1}(b)\right|$, we get $k=m$, i.e., $a$ and $b$ have the same number of terms.

Note that if we show $a_{m}=b_{m}$, Lemma 3.5 and the induction hypothesis would imply that $a$ and $b$ have the same corresponding 2 -free integer partition. On the contrary, let $a_{m} \neq b_{m}$. Without loss of generality we can assume that $a_{m}<b_{m}$. We will show that this implies that $\left|D_{n+a_{m}}(a)\right|<\left|D_{n+a_{m}}(b)\right|$, which is a contradiction to $a \stackrel{d}{\sim} b$

A constructive pair is a pair $(c, P)$ where $c=\left(c_{1}, \ldots, c_{l}\right)$ is a composition of $a_{m}$ and $P$ is a sequence that consists of the numbers $1,2, \ldots, l$ in order and $m$ boxes such that each box contains at most one of the numbers. To each such pair $(c, P)$ we associate the composition in $D_{n+a_{m}}(b)$ obtained by replacing each unboxed number $i$ in the sequence $P$ by $c_{i}$ and the $j^{t h}$ box by $b_{j}$ if it is empty and by $b_{j}+c_{i}$ if it contains the number $i$. We will call this composition $b(c, P)$.

Example 3.2. Suppose $b=(8,6,6,5), a_{m}=4, c=(1,2,1)$, and $P=1 \square \square 2 \square \square$. Then the composition $b(c, P)$ is $(1,8,8,1,6,5)$.

The construction of $b(c, P)$ from $b$ be thought of visually as follows: Think of any composition as towers of boxes. The boxes in the sequence $P$ can be thought of as the top view of $b$ and the number $i$ indicates where to add $c_{i}$ boxes to $b$. The construction in the example is shown in Figure 1.

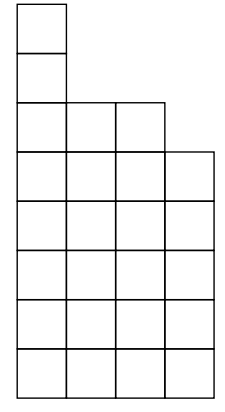

$b$

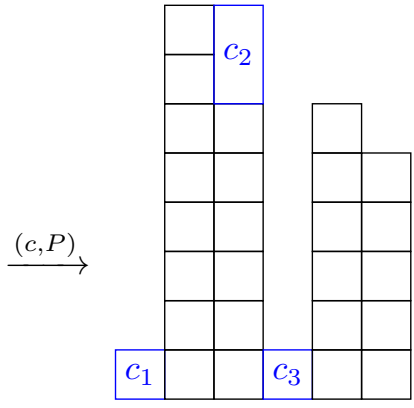

$b(c, P)$

Figure 1: Construction corresponding to the constructive pair given in Example 3.2

Using any occurrence of $b$ in a composition $b^{\prime}$ in $D_{n+a_{m}}(b)$, we can obtain a constructive pair $(c, P)$ such that $b(c, P)=b^{\prime}$. This is done as follows: Suppose $b^{\prime}=\left(b_{1}^{\prime}, \ldots, b_{p}^{\prime}\right)$ is a composition in $D_{n+a_{m}}(b)$ and $1 \leq$ $i(1)<i(2)<\cdots<i(m) \leq p$ is an occurrence of $b$. Construct $P$ from $b^{\prime}$ by first replacing $b_{j}^{\prime}$ by a ball if $j \notin\{i(1), \ldots, i(m)\}$. Then for any $j \in[m]$, replace $b_{i(j)}^{\prime}$ by an empty box if $b_{i(j)}^{\prime}=b_{j}$ and by a box containing a ball if $b_{i(j)}^{\prime}>b_{j}$. Suppose there are $l$ balls in $P$, we replace them by the numbers $1, \ldots, l$ in order. Now for each $i \in[l]$, 
1. set $c_{i}$ as $b_{j}^{\prime}$ if $i$ is an unboxed number and the $j^{\text {th }}$ term in $P$, and

2. set $c_{i}$ as $b_{i(j)}^{\prime}-b_{j}$ if $i$ is a boxed number and the $i(j)^{t h}$ term of $P$.

The above procedure might be more clear when compositions are thought of as towers of boxes. For example, in Figure 1, if the black boxes in $b(c, P)$ are used as the occurrence of $b$ in the above procedure, we obtain the constructive pair $(c, P)$ of Example 3.2.

It is clear that if $b^{\prime}=b(c, P)$ for some constructive pair $(c, P)$, then there is a canonical occurrence of $b$ in $b^{\prime}$ formed using the original copy of $b$ to which terms were added in the construction. Using this canonical occurrence in the above procedure we can get back the original constructive pair $(c, P)$. However, since $b$ has all terms strictly greater than $a_{m}$, there is a unique occurrence of $b$ in any composition $b^{\prime}$ in $D_{n+a_{m}}(b)$. Hence, there is a unique constructive pair $(c, P)$ such that $b(c, P)=b^{\prime}$ for any composition $b^{\prime}$ of $D_{n+a_{m}}(b)$. Therefore, the set $D_{n+a_{m}}(b)$ is in bijection with the set of constructive pairs.

Since $a$ also has $m$ terms, the same method can be used to associate a composition in $D_{n+a_{m}}(a)$ to a constructive pair $(c, P)$, and we call this composition $a(c, P)$. Just as for $b$, any composition $a^{\prime} \in D_{n+a_{m}}(a)$ can be obtained from $a$ using a constructive pair. However, $a\left(\left(a_{m}\right), P_{1}\right)=a\left(\left(a_{m}\right), P_{2}\right)$ where $P_{1}$ has 1 between the $(m-1)^{t h}$ and $m^{t h}$ box and $P_{2}$ has 1 after the $m^{t h}$ box. Hence we get $\left|D_{n+a_{m}}(a)\right|<\left|D_{n+a_{m}}(b)\right|$, which completes the proof.

\section{Acknowledgements}

We thank Priyavrat Deshpande for various helpful comments and suggestions. The second author is partially supported by a grant from the Infosys Foundation.

\section{References}

[1] J. Bloom and S. Elizalde, Pattern avoidance in matchings and partitions, Electron. J. Combin. 20(2) (2013), Article 5.

[2] W. Y. C. Chen, E. Y. P. Deng, R. R. X. Du, R. P. Stanley, and C. H. Yan, Crossings and nestings of matchings and partitions, Trans. Amer. Math. Soc. 359(4) (2007), 1555-1575.

[3] A. M. Goyt, Avoidance of partitions of a three-element set, Adv. in Appl. Math. 41(1) (2008), 95-114.

[4] V. Jelínek, T. Mansour, and M. Shattuck, On multiple pattern avoiding set partitions, Adv. in Appl. Math. 50(2) (2013), 292-326.

[5] M. Klazar, On abab-free and abba-free set partitions, European J. Combin. 17(1) (1996), 53-68.

[6] D. E. Knuth, The art of computer programming, Volume 1, Addison-Wesley, Reading, MA, 1997. Fundamental algorithms, Third edition.

[7] B. E. Sagan, Pattern avoidance in set partitions, Ars Combin. 94 (2010), 79-96.

[8] N. J. A. Sloane, The On-Line Encyclopedia of Integer Sequences, http://oeis.org. 